\title{
On Hard Decision Upper Bounds for Coded $M$-Ary Hierarchical Modulation
}

\author{
Suayb S. Arslan, Pamela C. Cosman and Laurence B. Milstein \\ Department of Electrical and Computer Engineering \\ University of California, San Diego, La Jolla, CA 92093-0407 \\ Email: \{sarslan, pcosman, lmilstein $\} @$ ucsd.edu
}

\begin{abstract}
Hierarchical modulation is used in conjunction with error correction coding in many multimedia communication systems to provide unequal protection. We show that hard decision upper bounds given for binary symmetric channels can be used to find upper bounds for the bit error rate of different bit classes of a coded $M$-ary hierarchical modulation.
\end{abstract}

\section{INTRODUCTION}

A LTHOUGH hard decision decoding generally provides inferior performance compared to soft decision decoding, it may be well suited for receivers that require low complexity implementation. For example, the IEEE 802.11a standard uses hard decision decoding to reduce the complexity of the receiver structure. Also, optical communications require error control codes with low decoding complexity, so hard decision decoding might be preferable [1]. In some cases, a receiver might be equipped with both hard decision and soft decision decoding capability. While more expensive in the initial outlay, such a dual decoder allows for savings on energy consumption any time hard decision is deemed good enough for current conditions [2].

Hierarchical modulation is very well suited for prioritized transmission of information of unequal significance. It is utilized to give unequal error protection (UEP) for High Priority (HP) and Low Priority (LP) bit classes. A hierarchical parameter characterizes the non-uniform spacing of the symbols in a hierarchical constellation. For example, for a hierarchical QAM constellation, the hierarchical parameter is sometimes defined as the ratio of the distance from the origin to an outer point to the distance from the origin to an inner point [3]. Hierarchical modulation is commonly used in conjunction with error correction coding in different communication systems, such as digital broadcast systems [4] as well as many other multimedia services.

Performance of a convolutional code is usually characterized in terms of the upper bounds for Bit Error Rate (BER). In [5], a simple hard decision bound is derived for block codes using hierarchical PSK modulation, and in [6], upper bounds for different protection classes of hierarchical-16QAM (H-16QAM) are derived. The main difference of our study is that we derive simpler upper bound expressions (similar in form to those in [7] and [8]) that will be applicable to a

This work was supported by Intel Inc., the Center for Wireless Communications (CWC) at UCSD, the UC Discovery Grant program of the state of California and National Science Foundation grant CCF-0915727.

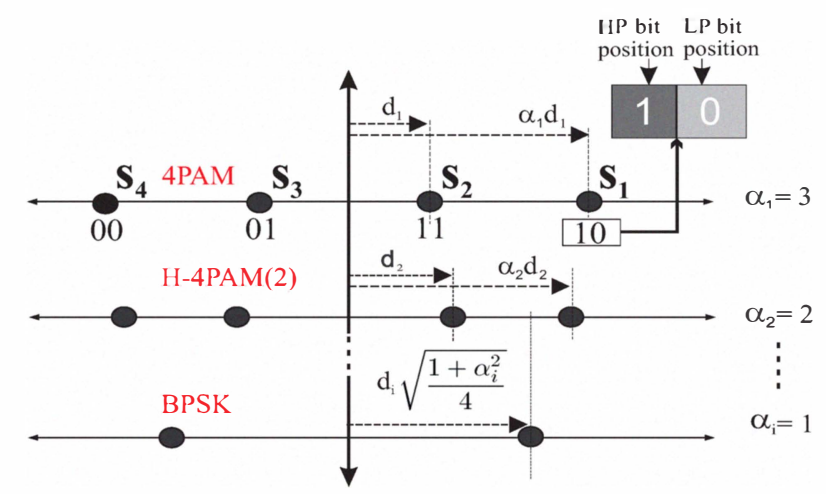

Fig. 1: H-4PAM provides two different levels of protection

wider class of hierarchical constellations under memoryless channel models. Our analysis is based on the observation that HP/LP bit positions experience different bit error probabilities based on the topology of the hierarchical constellation and the channel model. We prove that hard decision upper bounds given for convolutional codes for a BSC [7] can be used to compute the upper bounds for each bit class of the coded hierarchical modulation under AWGN and flat fading channels.

\section{Coded HierarchicAl MOdUlation AND THE PROBLEM FORMULATION}

Hierarchical modulation is utilized to give unequal transmission reliability to High Priority (HP) and Low Priority (LP) bit classes, and Fig. 1 illustrates a $\operatorname{H-} 4 \operatorname{PAM}(\alpha)$ constellation as an example. As shown in Fig. 1, hierarchical parameter $\alpha$ is the ratio of the distances of the two symbols to the origin on one side of a constellation. In going from one constellation to another, we vary $\alpha$ so that HP bits and LP bits will have different error probabilities. When $\alpha=1$, we have BPSK, and when $\alpha=3$, we have a conventional 4PAM constellation. Hierarchical modulation is often used in conjunction with error correction coding in multimedia communication systems. For example, different priority class source bit streams can be encoded using one of a class of punctured codes (such as Rate Compatible Punctured Convolutional (RCPC) codes [9]) for transmission of each priority class. This way, a gradual source degradation is achieved at the receiver.

Consider the H-4PAM constellation shown in Fig. 1 that consists of symbols $\left\{s_{1}, s_{2}, s_{3}, s_{4}\right\}$. Let us consider the HP bit of the binary representation of those symbols, and 
denote the error event $e_{i j}$ when bit $i \in\{0,1\}$ flips to bit $j \in\{0,1\}, i \neq j$. Let $\epsilon_{1} \triangleq P\left(e_{10} \mid s_{1}\right.$ is transmitted $)$, and $\epsilon_{2} \triangleq P\left(e_{10} \mid s_{2}\right.$ is transmitted $)$. Note that $P\left(e_{01} \mid s_{3}\right.$ is transmitted $)=\epsilon_{2}$ and $P\left(e_{01} \mid s_{4}\right.$ is transmitted $)=$ $\epsilon_{1}$. Thus, we can have different BERs for HP bits depending on the values of $\alpha$ and of the LP bits. Those BERs can easily be calculated for AWGN and flat independent Rayleigh channels as [10]

$\left(\epsilon_{1}, \epsilon_{2}\right)= \begin{cases}\text { AWGN: } & \left(Q\left(\sqrt{\frac{8 \gamma}{1+\alpha^{2}}}\right), Q\left(\sqrt{\frac{8 \gamma \alpha^{2}}{1+\alpha^{2}}}\right)\right) \\ \text { Flat Rayleigh: } & \left(\frac{1}{2}-\frac{1}{2} \sqrt{\frac{\lambda}{1+\lambda}}, \frac{1}{2}-\frac{1}{2} \sqrt{\frac{\alpha^{2} \lambda}{1+\alpha^{2} \lambda}}\right)\end{cases}$

where $\lambda=\frac{8 \sigma^{2} \gamma}{1+\alpha^{2}}, \gamma\left(=\frac{E_{b}}{N_{0}}\right)$ is the average SNR per bit, $\alpha$ is the hierarchical parameter, $\sigma$ is the parameter of the Rayleigh distribution and $Q(z)=\frac{1}{\sqrt{2 \pi}} \int_{z}^{\infty} e^{-\frac{x^{2}}{2}} d x$. We can also generate a similar set of error probabilities for LP bits.

In a given hierarchical constellation, suppose that class $i$ contains the symbols that correspond to an error probability of $\epsilon_{i}$ for the high priority bits (We could alternatively focus on the low priority bits). We define $p_{i}$ to be the probability of randomly drawing any symbol from class $i$. In this study, we only consider two classes, so we set $p_{1}=1-p$ and $p_{2}=p$ to simplify the notation. For example, in H-4PAM, we have $p=0.5$. Note that we can find constellations with $p \neq 0.5$. An example is shown in Fig. 2 where $\theta_{1}$ and $\theta_{2}$ are hierarchical parameters of two concentric hierarchical 2/4 PSK signals [3]. Consider the first (HP) bit position and let $\varsigma_{1}$ and $\varsigma_{2}$ be the radii of the concentric circles. If the symbols that have binary representations 001, 010 and 011 align vertically, as shown in Fig. 2 , then we have $d_{001}=d_{010}=d_{011}=\varsigma_{2} \cos \theta_{1}=\varsigma_{1} \sin \theta_{2}$ where $d_{001}, d_{010}$ and $d_{011}$ are their Euclidian distances to the decision boundary, respectively. Thus, if the BER of the first (HP) bit is $\epsilon_{2}$, when we send any of these three symbols, the BER will be $\epsilon_{1}, \epsilon_{1} \neq \epsilon_{2}$ when we send the remaining symbol on the right hand side of the constellation. Clearly, the same argument is valid when the HP bit is 1 . Therefore, for this constellation $p=0.75$. In general, $p$ is determined by the topology of the constellation for memoryless channels.

A 2-layer transmission system block diagram is depicted in Fig. 3. After channel coding, coded priority bit streams are multiplexed so that the modulator maps the HP bit streams strictly to HP bit classes and the LP bit streams to LP bit classes in the constellation. The receiver coherently demodulates and makes hard decisions based on the received symbol stream. Then, the demodulated bit stream is demultiplexed into two priority classes. Finally, each priority class bit stream is fed to corresponding Maximum Likelihood (ML) decoders for independent decoding.

In this study, we start with Equation (1) from [9] below, and derive an expression for $P_{d^{(\beta)}}$ for coded hierarchical modulations. We show that the $\operatorname{BER}\left(P_{e}\right)$ for the HP bits (or LP bits) can be upper bounded by

$$
P_{e} \leq \frac{1}{\delta} \sum_{d^{(\beta)}=d_{f}}^{\infty} c_{d^{(\beta)}} P_{d^{(\beta)}}
$$

where $\delta$ is the puncturing period, $c_{d^{(\beta)}}$ is the coefficient of the bit Input Weight Enumeration Function (IWEF) of a given

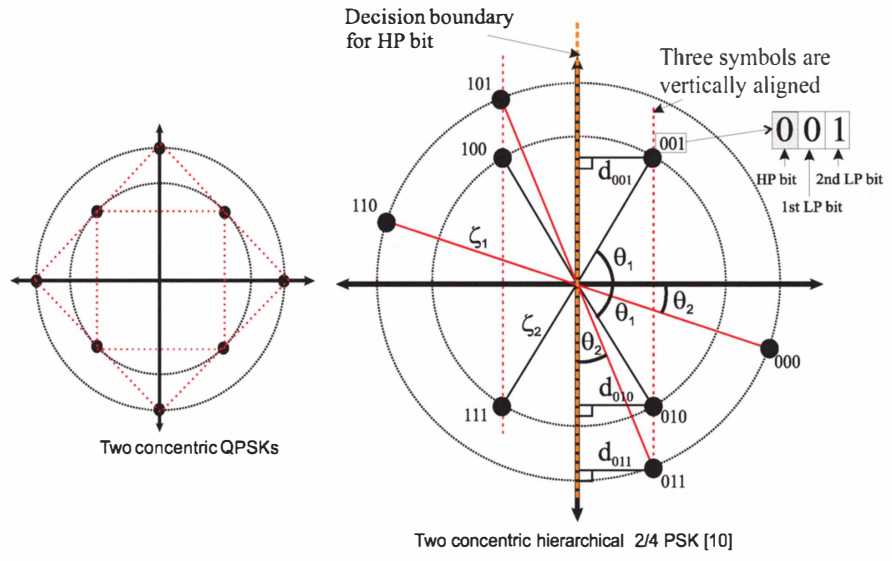

Fig. 2: A hierarchical constellation where we have $p=0.75$.

code $\beta, d_{f}$ is the free distance of the code, and $P_{d^{(\beta)}}$ is the probability of selecting an incorrect path in the trellis $\mathcal{T}$. Note $\delta=1$ for unpunctured convolutional codes. Throughout the paper, BER refers to the decoded bit error rate rather than raw bit error rate.

\section{HARD DECISION UPPER BOUND}

Assume that the all-zero binary sequence is transmitted and all the bits are going through $B S C(\epsilon)$. Now suppose that the path in $\mathcal{T}$ being compared with the all-zero path at some node has distance $n$ from the all-zero path. The probability of selecting the incorrect path is given by

$$
P_{n}=\sum_{k=\frac{n+1}{2}}^{n}\left(\begin{array}{l}
n \\
k
\end{array}\right) \epsilon^{k}(1-\epsilon)^{n-k}
$$

Now, for simplicity, assume that we have two different symbol groups that corresponds to raw bit error rates of $\epsilon_{1}$ and $\epsilon_{2}$. From these $n$ bit positions, say we have $s$ positions that have a bit error probability $\epsilon_{1}$, and $n-s$ positions that have a bit error probability $\epsilon_{2}$. Then, the average probability of selecting the wrong path in $\mathcal{T}$ is given by

$$
P_{n}=\sum_{s=0}^{n} \operatorname{Pr}\{s ; n\} \psi_{s}\left(k \geq \frac{n+1}{2}\right)
$$

where $\operatorname{Pr}\{s ; n\}$ is the probability of having exactly $s$ bit positions that have the bit error probability $\epsilon_{1}$, and $\psi_{s}\left(k \geq \frac{n+1}{2}\right)$

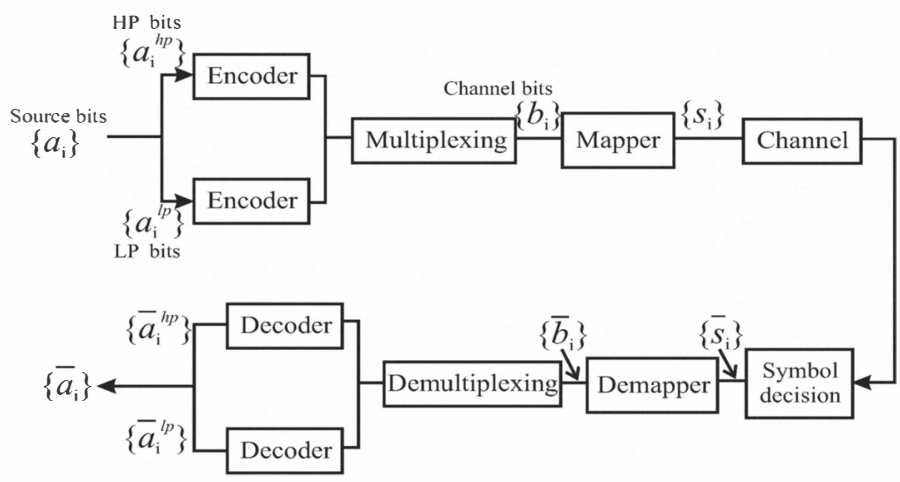

Fig. 3: System block diagram for 2-layer transmission 

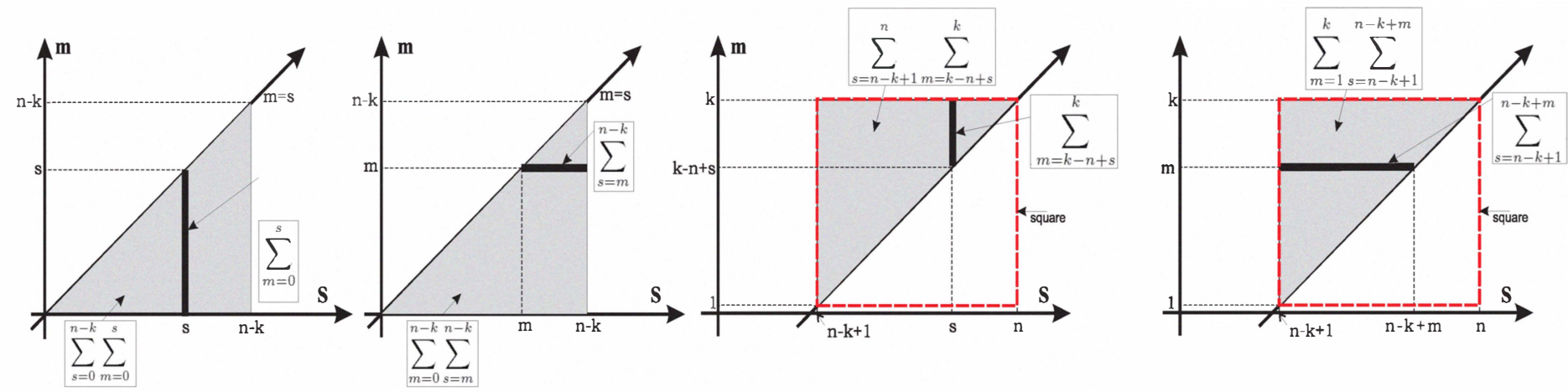

Fig. 4: Double sums in (8) and (9) are equivalent.

is the probability of selecting an incorrect path when exactly $s$ positions have the bit error probability $\epsilon_{1}$. Note that

$$
\operatorname{Pr}\{s ; n\}=\left(\begin{array}{l}
n \\
s
\end{array}\right)(1-p)^{s} p^{n-s}
$$

Lemma $\psi_{s}\left(k \geq \frac{n+\kappa}{2}\right)$ is given by (5) with $\kappa \in\{1,2\}$.

Proof For a given distance $n$ path i.e., the distance to the all-zero path is $n$, assume that $s$ bit positions have the bit error probability $\epsilon_{1}$ and $n-s$ positions have bit error probability $\epsilon_{2}$. Now assume $k$ positions are in error. Of these $k$ positions, assume that $m$ positions are chosen from the bits that have a bit error probability $\epsilon_{1}$ and $k-m$ positions are chosen from the bits that have a bit error probability $\epsilon_{2}$. The probability of this event happening is given by $\left(\begin{array}{c}s \\ m\end{array}\right) \epsilon_{1}^{m}\left(1-\epsilon_{1}\right)^{s-m}\left(\begin{array}{c}n-s \\ k-m\end{array}\right) \epsilon_{2}^{k-m}\left(1-\epsilon_{2}\right)^{n-s-k+m}$. We now need to sum over $m$ and $k$. Note that $\left(\begin{array}{c}s \\ m\end{array}\right)$ and $\left(\begin{array}{c}n-s \\ k-m\end{array}\right)$ are nonzero when $0 \leq m \leq s$ and $0 \leq k-m \leq n-s$ and zero otherwise. We have $m \leq k$, because $k$ is the total number of errors . Therefore $m \leq s, m \leq k \Rightarrow m \leq \min \{s, k\}$ and $k-m \leq n-s, m \geq 0 \Rightarrow\{m \geq k-n+s, m \geq 0\} \Rightarrow\{m \geq$ $\left.[k-n+s]^{+}\right\}$. Also, since we assume the all-zero path is sent, for the selected path to be in error we need to have $k \geq \frac{n+1}{2}$ for odd $n$ and $k \geq \frac{n}{2}+1$ for even $n$.

If we plug (4) and (5) in (3), we can write the sequence of equations from (6) to (11). The explanation for each step is given as follows:

- (7) to (8): The total sum is divided into two double sums. The first double sum assumes $s \leq n-k<k$. Therefore $\min \{s, k\}=s$. Also $[k-n+s]^{+}=0$ for $s \leq n-k$. The second double sum assumes $s \geq n-k+1$. If $k \leq s$, then we have $\min \{s, k\}=k$. Otherwise, we have $s<k$. Since $\left(\begin{array}{l}a \\ b\end{array}\right)=0$ if $b>a$, if we sum $m$ up to $k$ instead of up to $s$, we will be adding $k-s$ zeros, so the sum will not change. Also, $[k-n+s]^{+}>0$ for $s \geq n-k+1$. We show the equivalence of the double sums in the second inequality of (8) by plotting the two dimensional area that

$$
\psi_{s}\left(k \geq \frac{n+\kappa}{2}\right)=\sum_{k=\frac{n+\kappa}{2}}^{n} \sum_{m=[k-n+s]^{+}}^{\min \{k, s\}}\left(\begin{array}{c}
s \\
m
\end{array}\right) \epsilon_{1}^{m}\left(1-\epsilon_{1}\right)^{s-m}\left(\begin{array}{c}
n-s \\
k-m
\end{array}\right) \epsilon_{2}^{k-m}\left(1-\epsilon_{2}\right)^{n-s-k+m},[x]^{+}=\left\{\begin{array}{ll}
x & \text { if } x \geq 0, \\
0 & \text { if } x<0
\end{array} \text { and } \kappa=\left\{\begin{array}{l}
1 \quad \text { if } n \text { odd } \\
2 \quad \text { if } n \text { even }
\end{array}(5)\right.\right.
$$

$$
\begin{aligned}
& P_{n}=\sum_{s=0}^{n}\left(\begin{array}{l}
n \\
s
\end{array}\right)(1-p)^{s} p^{n-s} \sum_{k=\frac{n+1}{2}}^{n} \sum_{m=[k-n+s]^{+}}^{\min \{k, s\}}\left(\begin{array}{c}
s \\
m
\end{array}\right) \epsilon_{1}^{m}\left(1-\epsilon_{1}\right)^{s-m}\left(\begin{array}{c}
n-s \\
k-m
\end{array}\right) \epsilon_{2}^{k-m}\left(1-\epsilon_{2}\right)^{n-s-k+m} \\
& =\sum_{k=\frac{n+1}{2}}^{n} \sum_{s=0}^{n} \sum_{m=[k-n+s]}^{\min \{k, s\}} \underbrace{(1-p)^{s} p^{n-s}\left(\begin{array}{l}
n \\
s
\end{array}\right)\left(\begin{array}{c}
s \\
m
\end{array}\right) \epsilon_{1}^{m}\left(1-\epsilon_{1}\right)^{s-m}\left(\begin{array}{c}
n-s \\
k-m
\end{array}\right) \epsilon_{2}^{k-m}\left(1-\epsilon_{2}\right)^{n-s-k+m}}_{\Lambda_{k, s, m}} \\
& =\sum_{k=\frac{n+1}{2}}^{n}\left(\sum_{s=0}^{n-k} \sum_{m=0}^{s} \Lambda_{k, s, m}+\sum_{s=n-k+1}^{n} \sum_{m=k-n+s}^{k} \Lambda_{k, s, m}\right)=\sum_{k=\frac{n+1}{2}}^{n}\left(\sum_{m=0}^{n-k} \sum_{s=m}^{n-k} \Lambda_{k, s, m}+\sum_{m=1}^{k} \sum_{s=n-k+1}^{n-k+m} \Lambda_{k, s, m}\right) \\
& =\sum_{k=\frac{n+1}{2}}^{n}\left(\sum_{m=0}^{n-k} \sum_{s=m}^{n-k} \Lambda_{k, s, m}+\sum_{m=n-k+1}^{k} \sum_{s=m}^{n-k} \Lambda_{k, s, m}+\sum_{m=1}^{k} \sum_{s=n-k+1}^{n-k+m} \Lambda_{k, s, m}+\sum_{s=n-k+1}^{n-k} \Lambda_{k, s, m}\right) \\
& =\sum_{k=\frac{n+1}{2}}^{n}\left(\sum_{m=0}^{k} \sum_{s=m}^{n-k} \Lambda_{k, s, m}+\sum_{m=0}^{k} \sum_{s=n-k+1}^{n-k+m} \Lambda_{k, s, m}\right) \\
& =\sum_{k=\frac{n+1}{2}}^{n} \sum_{m=0}^{k} \sum_{s=m}^{n-k+m}(1-p)^{s} p^{n-s}\left(\begin{array}{l}
n \\
s
\end{array}\right)\left(\begin{array}{c}
s \\
m
\end{array}\right) \epsilon_{1}^{m}\left(1-\epsilon_{1}\right)^{s-m}\left(\begin{array}{c}
n-s \\
k-m
\end{array}\right) \epsilon_{2}^{k-m}\left(1-\epsilon_{2}\right)^{n-s-k+m} \text { where }\left(\begin{array}{l}
a \\
b
\end{array}\right)=0 \text { if } b>a
\end{aligned}
$$


each double sum covers. This is shown in Fig. 4.

- (8) to (9): We added extra 0s to complete the sums. For the first double sum, we have $s \leq n-k$. Thus, extending the maximum value of $m$ from $n-k$ to $k$ will not make a difference because $\max \{s\}=n-k$. The same argument applies to the second double sum. Also, $\sum_{i=a}^{b} g(i)=0$ for $a>b$ where $g(i)$ is any function of $i$.

- (9) to (10): We combine the double sums.

A. $n$ odd

Theorem 1.A: $\forall \mathfrak{p} \in \mathbb{N}^{+}, n=2 \mathfrak{p}+1$, we have $P_{n}=\widetilde{P}_{n}$ where

$$
\begin{aligned}
\widetilde{P}_{n}=\sum_{k=\frac{n+1}{2}}^{n}\left(\begin{array}{l}
n \\
k
\end{array}\right)\left((1-p) \epsilon_{1}+p \epsilon_{2}\right)^{k} & \left(1-\left((1-p) \epsilon_{1}+p \epsilon_{2}\right)\right)^{n-k}
\end{aligned}
$$

This is the same expression given in (2), except that we replace $\epsilon$ with $(1-p) \epsilon_{1}+p \epsilon_{2}=\epsilon_{1}-p\left(\epsilon_{1}-\epsilon_{2}\right)$.

Proof By expanding (12), we obtain

$$
\begin{aligned}
\widetilde{P}_{n}=\sum_{k=\frac{n+1}{2}}^{n}\left(\begin{array}{l}
n \\
k
\end{array}\right) & \left.(1-p) \epsilon_{1}+p \epsilon_{2}\right)^{k} \\
& \left((1-p)\left(1-\epsilon_{1}\right)+p\left(1-\epsilon_{2}\right)\right)^{n-k}
\end{aligned}
$$

If we use the binomial expansion for (13), we obtain (14). Let us make the change of variables $z=s-m$ i.e., $s=$ $z+m$. Since $z \in[0, n-k]$, then $s \in[m, n-k+m]$, so we will have (15). This equals $P_{n}$ in (11), since $\left(\begin{array}{l}n \\ k\end{array}\right)\left(\begin{array}{l}k \\ m\end{array}\right)\left(\begin{array}{l}n-k \\ s-m\end{array}\right)=$ $\left(\begin{array}{l}n \\ s\end{array}\right)\left(\begin{array}{c}s \\ m\end{array}\right)\left(\begin{array}{c}n-s \\ k-m\end{array}\right)$.

\section{B. $n$ even}

Theorem 1.B: $\forall \mathfrak{p} \in \mathbb{N}, n=2 \mathfrak{p}$, we have $P_{n}=\widetilde{P}_{n}$.

Proof If $n$ is even, we have $k \geq \frac{n}{2}+1$. An incorrect path is chosen when the number of errors exceeds $\frac{n}{2}$. If it equals $\frac{n}{2}$, the decoder selects one of the paths randomly. Thus, with $\kappa=2$, we have

$$
P_{n}=\sum_{s=0}^{n} \operatorname{Pr}\{s ; n\} \psi_{s}\left(k \geq \frac{n}{2}+1\right)+\frac{1}{2} \mathfrak{P}(n / 2)
$$

where the $1 / 2$ comes from the fact that half of the time the decoder incurs an error and $\mathfrak{P}(n / 2)$ is the probability of selecting an incorrect path when the number of errors equals $n / 2$. Of these $n / 2$ bit positions, suppose $m$ bit positions have a bit error probability $\epsilon_{1}$, and for the remaining $n / 2$ bit positions, $z$ bit positions have a bit error probability $\epsilon_{1}$. The probability of this event happening is given by

$$
\left(\begin{array}{c}
n / 2 \\
m
\end{array}\right) \epsilon_{1}^{m} \epsilon_{2}^{\frac{n}{2}-m}\left(\begin{array}{c}
n / 2 \\
z
\end{array}\right)\left(1-\epsilon_{1}\right)^{z}\left(1-\epsilon_{2}\right)^{\frac{n}{2}-z}
$$

Let $\phi_{m, z}$ be the conditional probability of choosing a particular $n / 2$ bit locations given that $m+z$ bit positions have bit error probability $\epsilon_{1}$ as described above. Thus, in order to find an expression for $\mathfrak{P}(n / 2)$, we sum over $m$ and $z$ as follows:

$\mathfrak{P}(n / 2)=\sum_{m=0}^{n / 2} \sum_{z=0}^{n / 2} \phi_{m, z}\left(\begin{array}{c}n / 2 \\ m\end{array}\right) \epsilon_{1}^{m} \epsilon_{2}^{\frac{n}{2}-m}\left(\begin{array}{c}n / 2 \\ z\end{array}\right)\left(1-\epsilon_{1}\right)^{z}\left(1-\epsilon_{2}\right)^{\frac{n}{2}-z}$

where $\phi_{m, z}$ is given by

$$
\phi_{m, z}=\left(\begin{array}{c}
n \\
n / 2
\end{array}\right)(1-p)^{z+m} p^{n-z-m}
$$

Therefore, using the binomial expansion as shown in Theorem 1.A, we can write the second term in (16) as follows

$$
\begin{aligned}
\frac{1}{2} \mathfrak{P}(n / 2)=\frac{1}{2}\left(\begin{array}{c}
n \\
n / 2
\end{array}\right)( & \left.(1-p) \epsilon_{1}+p \epsilon_{2}\right)^{n / 2} \\
& \left(1-\left((1-p) \epsilon_{1}+p \epsilon_{2}\right)\right)^{n / 2}
\end{aligned}
$$

Since the first term in (16) follows the same line of proof shown for $\kappa=1$ (i.e., $n$ is odd and $k \geq \frac{n+1}{2}$ ), the details are omitted for the $\kappa=2$ case.

Therefore, $\forall d^{(\beta)} \in \mathbb{N}^{+}$, a more compact expression for $P_{d^{(\beta)}}$ in Equation (1) is given by

$$
\begin{aligned}
P_{d^{(\beta)}}= & \sum_{k=} \frac{d^{(\beta)}+1+\left[d^{(\beta)}+1\right]_{2}}{2}\left(\begin{array}{c}
d^{(\beta)} \\
k
\end{array}\right)\left(\epsilon_{0}\right)^{k}\left(1-\epsilon_{0}\right)^{d^{(\beta)}-k} \\
& +\frac{\left[d^{(\beta)}+1\right]_{2}}{2}\left(\begin{array}{c}
d^{(\beta)} \\
d^{(\beta)} / 2
\end{array}\right)\left(\epsilon_{0}\right)^{d^{(\beta)} / 2}\left(1-\epsilon_{0}\right)^{d^{(\beta)} / 2}
\end{aligned}
$$

where []$_{2}$ is modulo two equivalent of the argument and $\epsilon_{0}=$ $\epsilon_{1}-p\left(\epsilon_{1}-\epsilon_{2}\right)$.

\section{Numerical RESUlts AND CONCLUSiONS}

Throughout this section, we use two types of codes: (1) RCPC code with memory $M=6$ and $M=4$ given in [9], (2) NASA standard code [12]: $(7,1 / 2)$ convolutional code, used also in DVB-T [4].

We tested both AWGN and slowly varying flat Rayleigh fading channels using various hierarchical constellations: $\mathrm{H}$ 4PAM, H-16QAM, two concentric hierarchical 2/4 PSK [3]. In Table I, we show several parameters used in evaluating the proposed bound for these modulation formats. Note that $\epsilon_{1}$ and $\epsilon_{2}$ can be obtained for any channel and any priority bit level for a given constellation topology. In short, for the given simulation parameters, as long as we find the triple $\left(\epsilon_{1}, \epsilon_{2}, p\right)$, we can calculate the upper bound.

For punctured convolutional codes, the upper bound is given by Inequality (1). As noted, using the corresponding $c_{d^{(\beta)}}$, with

$$
\begin{aligned}
\widetilde{P}_{n} & =\sum_{k=\frac{n+1}{2}}^{n}\left(\begin{array}{l}
n \\
k
\end{array}\right) \sum_{m=0}^{k}\left(\begin{array}{l}
k \\
m
\end{array}\right)(1-p)^{m} \epsilon_{1}^{m} p^{k-m} \epsilon_{2}^{k-m} \sum_{z=0}^{n-k}\left(\begin{array}{c}
n-k \\
z
\end{array}\right)(1-p)^{z}\left(1-\epsilon_{1}\right)^{z} p^{n-k-z}\left(1-\epsilon_{2}\right)^{n-k-z} \\
& =\sum_{k=\frac{n+1}{2}}^{n} \sum_{m=0}^{k} \sum_{s=m}^{n-k+m}(1-p)^{s} p^{n-s}\left(\begin{array}{l}
n \\
k
\end{array}\right)\left(\begin{array}{l}
k \\
m
\end{array}\right) \epsilon_{1}^{m} p^{k-m} \epsilon_{2}^{k-m}\left(\begin{array}{c}
n-k \\
s-m
\end{array}\right)\left(1-\epsilon_{1}\right)^{s-m}\left(1-\epsilon_{2}\right)^{n-k-s+m}
\end{aligned}
$$




\begin{tabular}{|c|c|c|c|c|c|}
\hline Modulation & Priority & Channel & $\epsilon_{1}$ & $\epsilon_{2}$ & $p$ \\
\hline \hline H-4PAM/H-16QAM & HP & AWGN & $Q\left(\sqrt{\frac{8 \gamma}{1+\alpha^{2}}}\right)$ & $Q\left(\sqrt{\frac{8 \gamma \alpha^{2}}{1+\alpha^{2}}}\right)$ & 0.5 \\
\hline H-4PAM/H-16QAM & HP & Rayleigh & $\frac{1}{2}-\frac{1}{2} \sqrt{\frac{\lambda}{1+\lambda}}$ & $\frac{1}{2}-\frac{1}{2} \sqrt{\frac{\alpha^{2} \lambda}{1+\alpha^{2} \lambda}}$ & 0.5 \\
\hline Concentric H-2/4PSK & HP & AWGN & $Q\left(\varsigma_{1} \cos \theta_{1} \sqrt{3 \gamma}\right)[5]$ & $Q\left(\varsigma_{2} \cos \theta_{2} \sqrt{3 \gamma}\right)[5]$ & 0.75 \\
\hline
\end{tabular}

Table I: Parameters used in the evaluation of the bound for HP bits for different channel and constellation assumptions.

\begin{tabular}{|l|l|l|l|l|l|}
\hline Code & Rate & M & GP & $\delta$ & $c_{d}$ \\
\hline CC & $1 / 2$ & 6 & {$[171,131]$} & 1 & $c_{10}=36, c_{11}=0, \ldots$ \\
RCPC & $8 / 9$ & 6 & {$[133,171,65]$} & 8 & $c_{3}=24, c_{4}=740, \ldots$ \\
RCPC & $2 / 3$ & 6 & {$[133,171,65]$} & 8 & $c_{6}=12, c_{7}=280, \ldots$ \\
RCPC & $1 / 2$ & 4 & {$[13,29,17,27]$} & 8 & $c_{7}=32, c_{8}=96, \ldots$ \\
RCPC & $2 / 3$ & 4 & {$[13,29,17,27]$} & 8 & $c_{4}=4, c_{5}=0, \ldots$ \\
RCPC & $2 / 5$ & 4 & {$[13,29,17,27]$} & 8 & $c_{8}=2, c_{9}=34, \ldots$ \\
\hline
\end{tabular}

Table II: Parameters of the codes. GP: Generator polynomial.

$\delta=1$, upper bounds for unpunctured convolutional codes can also be calculated using (1). It is enough to sum a finite number of terms in (1) to get close bounds [9]. IWEFs of some of the convolutional codes used in our simulations are summarized in Table II.

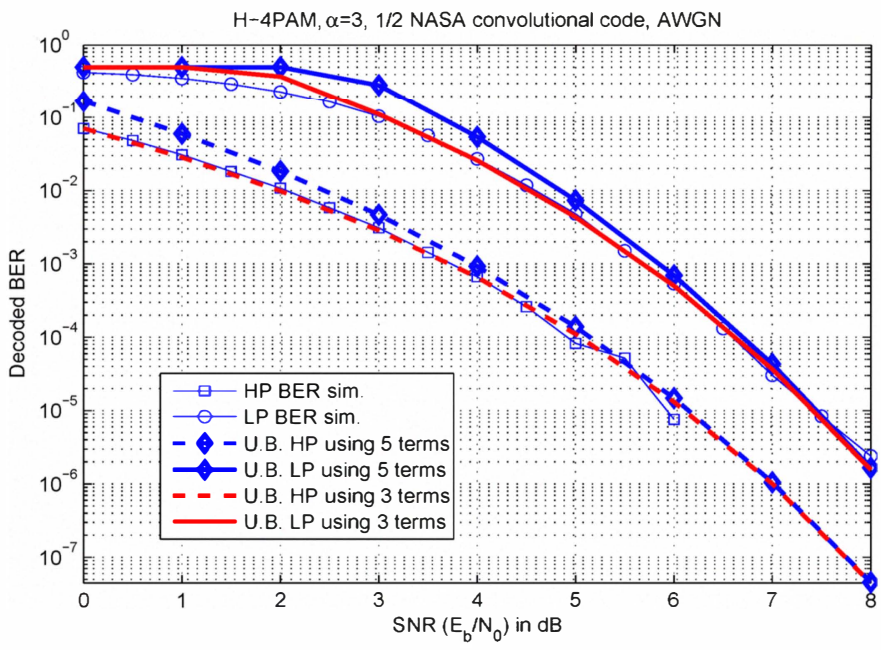

Fig. 5: Conventional 4PAM $(\alpha=3)$ coded with NASA standard code.

Suppose that $L$ denotes the number of priority layers that a hierarchical modulation can support. The first simulation assumes an AWGN channel using a conventional H-4PAM $(L=2)$ constellation with a single hierarchical parameter $\alpha=3$ (i.e., non-hierarchical modulation). A Monte Carlo simulation result using NASA's $1 / 2$ convolutional code and $\mathrm{H}$ 4PAM as well as the upper bounds suggested in this paper are illustrated in Fig. 5. We have shown upper bounds using both the first three and the first five terms in (1). Next, in Fig. 6, a rate $8 / 9$ RCPC code with memory $M=6$ is used with various $\alpha$ values using H-16QAM with $L=2$. Note that inphase and quadrature components of a given H-16QAM can be thought of as two independent H-4PAM constellations. We have used only the first 4 terms in (1) while evaluating the bounds. Fig. 6 suggests that the bounds give close approximations to the simulation results for the SNRs of interest over a reasonable range of the hierarchical parameter set.

Let us now consider the two concentric 2/4 PSK waveforms

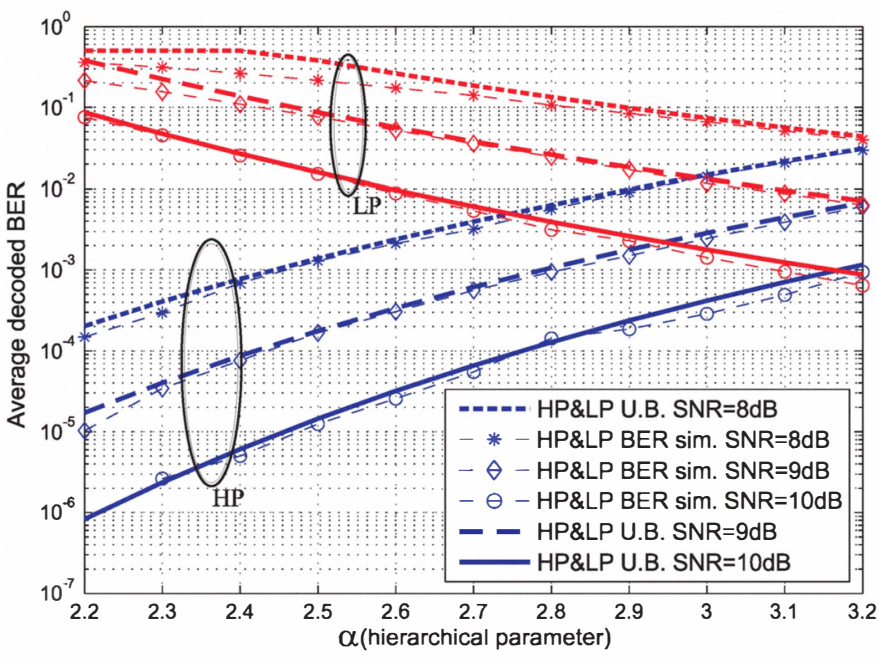

Fig. 6: Hierarchical 16QAM coded with RCPC 8/9 code with $M=6$ from [9]. Bold curves are the upper bounds. U.B. : Upper Bound.

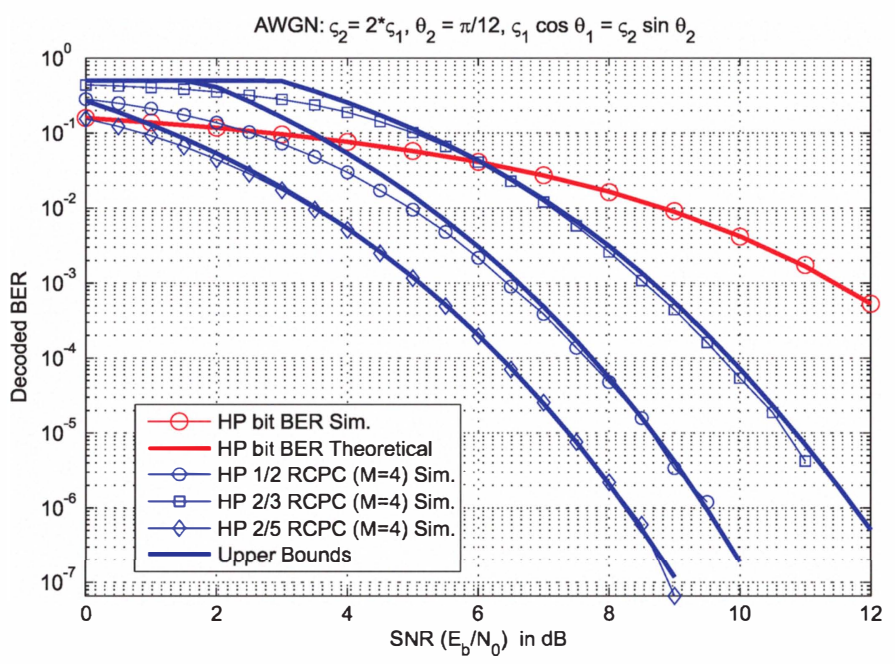

Fig. 7: Two concentric 2/4 PSKs coded with several RCPC codes with $M=4$ from [9].

$(L=3)$ coded with RCPC codes with memory $\mathrm{M}=4$ from [9]. We choose $\theta_{2}=\pi / 12$ and $\varsigma_{2}=2 \times \varsigma_{1}$. Therefore, we have $\theta_{1}=\arccos \left(2 \sin \frac{\pi}{12}\right)$. Let us consider only the HP bit location and the associated information BER in AWGN. Fig. 7 shows the simulation as well as the suggested upper bounds, based on the information summarized in Table I. It also shows the uncoded case both using simulations and the theoretical result, which are in close agreement. Finally, we consider a flat Rayleigh fading channel using a conventional 16QAM constellation. A Monte Carlo simulation result using a 2/3 RCPC code with $\mathrm{M}=6$ and $\mathrm{H}-16 \mathrm{QAM}$, as well as the bounds, are illustrated in Fig. 8. 


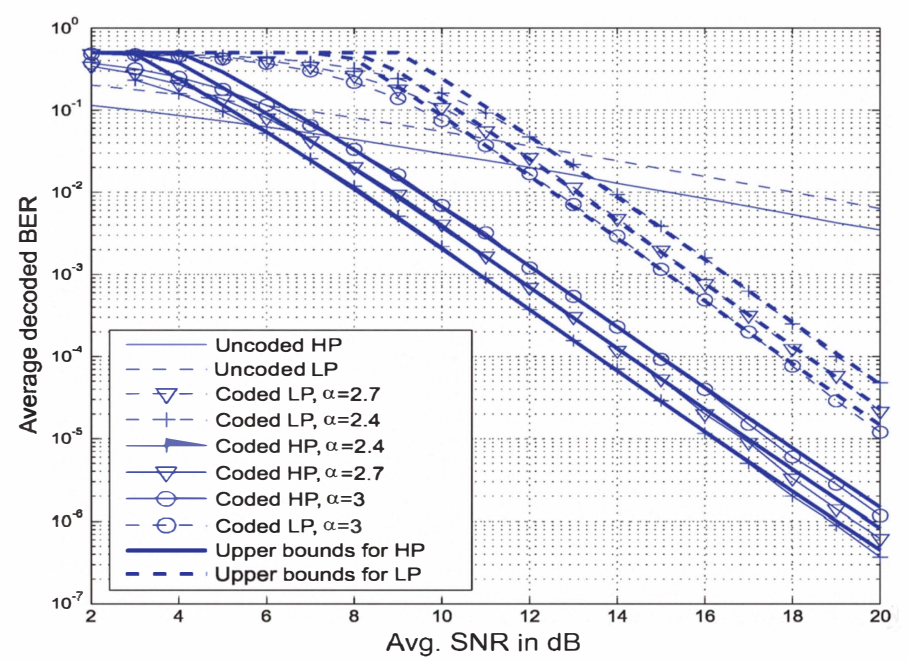

Fig. 8: RCPC $2 / 3$ code used in conjunction with H-16QAM under flat Rayleigh fading channel.

In conclusion, we extended the results found in [7] to coded hierarchical modulation. We considered a particular case by considering $M$-ary hierarchical constellations with two symbol groups giving different bit error probabilities for each priority level bit stream. Bounds show a good approximation to the numerical results for the coded hierarchical modulation under both AWGN and flat Rayleigh fading channels.

\section{REFERENCES}

[1] S.M. Tseng, M.C. Chang, H.L. Chan, Y.T. Hsu, "A Simplified Decoding Scheme of an Optical Transform Domain Error Control Code" Journal of optical communications, vol. 27, No 4, pp. 238-240, 2006.

[2] P.F. Swaszek and W. Jones, "How often is hard-decision decoding enough?", IEEE Trans. Inform. Theory, vol. 44, pp. 1187-1193, May 1998.

[3] P.K. Vitthaladevuni and M.S. Alouini, "Exact BER Computation of Generalized Hierarchical PSK Constellations," IEEE Trans. on Commun., vol. 51, No. 12, pp. 2030-2037, Dec. 2003.

[4] ETSI, EN 300 744, V1.5.1, Digital Video Broadcasting (DVB): framing structure, channel coding and modulation for digital terrestrial television, Nov. 2004

[5] P.K. Vitthaladevuni and M.S. Alouini, "An upper bound on the BER of block coded hierarchical constellations," 2003 IEEE Pacific Rim Conference on. Communications, Computers and Signal Processing (PACRIM 03), vol. 2, pp. 950-953. Aug. 2003.

[6] J.H. Lim and S.B. Gelfand, "Performance analysis of hierarchical coded modulation systems," Global Telecommunications Conference, 2000.

[7] A.J. Viterbi, "Convolutional codes and their performance in communication systems," IEEE Trans. on Commun. Technol. vol. COM-19, pp. 751-771, 1971

[8] J.Hagenauer, "Viterbi decoding of convolutional codes for fading- and burst-channels," Proc. of the 1980 Zurich Seminar on Digital Communications, pp. G2.1 - G2.7., 1980

[9] J. Hagenauer, "Rate-Compatible Punctured Convolutional Codes (RCPC Codes) and Their Applications," IEEE Trans. on Commun., vol. 36, No. 4, pp. 389-400, April 1997.

[10] PK. Vitthaladevuni and M.S. Alouini, "BER Computation of 4/M. QAM Hierarchical Constellations," IEEE Trans. on Broaakasting, vol. 47, no. 3, pp.228-239, Sept 2001.

[11] J.G. Proakis, "Digital Communications," 3rd edition, New York: McGraw Hill, 1995.

[12] R.E. Edelson, B.D. Madsen, E.K. Davis and G.W. Garrison, Voyager telecommunications: the broadcast from Jupiter, Science 204, 913-921 (1979).

[13] S. Lin and D. J. Costello Jr., "Error Control Coding", Second Edition, Prentice Hall, 2004 